\title{
An Analytical Solution to Neumann-Type Mixed Boundary Poiseuille Microfluidic Flow in Rectangular Channel Cross-Sections (Slip/No-Slip) including a Numerical Technique to Derive It
}

\author{
Christiane Richter, Frederik Kotz, N. Keller, Tobias M. Nargang, Kai Sachsenheimer, \\ Dorothea Helmer, Bastian E. Rapp*
}

Karlsruhe Institute of Technology (KIT), Istitute of Microstructure Technology (IMT), Eggenstein-Leopoldshafen, Germany

Email: bastian.rapp@kit.edu

How to cite this paper: Richter, C., Kotz, F., Keller, N., Nargang, T.M., Sachsenheimer, K., Helmer, D. and Rapp, B.E. (2017) An Analytical Solution to Neumann-Type Mixed Boundary Poiseuille Microfluidic Flow in Rectangular Channel Cross-Sections (Slip/No-Slip) including a Numerical Technique to Derive It. J. Biomedical Science and Engineering, 10, 205-218. https://doi.org/10.4236/jbise.2017.105016

Received: February 20, 2017

Accepted: May 13, 2017

Published: May 16, 2017

Copyright (c) 2017 by authors and Scientific Research Publishing Inc. This work is licensed under the Creative Commons Attribution International License (CC BY 4.0).

http://creativecommons.org/licenses/by/4.0/

\begin{abstract}
In most microfluidic applications, pressure-driven Poiseuille flow in a contained cross-section with no-slip boundary conditions is the underlying fluidmechanical model. Solutions for this problem exist for many known crosssections. We have recently demonstrated a simple method to solve the relevant Poisson equation using a finite difference scheme in a spreadsheet analysis tool such as Microsoft Excel. The numerical solutions obtained from such a spreadsheet are close-to-exact to the analytical solutions with errors on the order of only a few percent. However, there are numerous applications in microfluidics for which the no-slip boundary condition is not valid. Examples include drag-reducing air-retaining surfaces as well as open-channel flow. For these scenarios few to no analytical models exist. In this paper, we derive an analytical model for mixed boundary conditions (slip/no-slip) in two dimensions in a rectangular channel cross-section. We also demonstrate that the equivalent numerical solution can be derived conveniently by adaption of the spreadsheet. In general, mixed boundary-type flow scenarios are especially difficult to solve analytically whereas numerical solutions can be derived using Microsoft Excel within seconds.
\end{abstract}

\section{Keywords}

Microfluidics, Numerics, Navier-Stokes Equation, Slip Condition, No-Slip Condition

\section{Introduction}

Many effects in microfluidics rely on the sound understanding of the underlying 
fluid mechanics of the flow. Compared to macroscopic and high Reynoldsnumber flows, the flow cases in microfluidics are usually significantly simpler due to the fact that most microfluidic applications are within the strictly laminar flow regime and the flow is assumed to be parallel. In addition, assuming a flow to be stationary and fully-developed allows dropping additional terms of the Navier-Stokes equation. The remaining, simplified version of the Navier-Stokes equation is a Poisson equation. As we have demonstrated recently, this equation can be solved using a numerical scheme based on a finite difference approach. This approach can be implemented conveniently in a spreadsheet analysis tool such as Microsoft Excel [1]. We have shown that the numerical solutions derived compare well to the analytical solutions for various flow cases including the flow in circular cross-sections (Hagen-Poiseuille flow) as well as in rectangular channel profiles. One of the most important points to consider when implementing a numerical scheme is the behaviour of the boundaries of the computational domain. In all of the cases discussed the flow was assumed to exhibit no-slip behaviour on the boundary, i.e., the flow velocity on the boundaries was assumed to be zero. This type of boundary condition is referred to as Dirichlet-type boundary condition where the value of the dependent variable (in this case the flow velocity $v_{x}$ parallel to the axis of the channel) on the boundary is assumed to have a distinct value. However, many cases in microfluidics require Neumann-type boundary condition where the gradient of the dependent variable, i.e., the first derivative of the velocity profile, is assumed to obtain a certain value. The most important cases of Neumann-type boundary conditions are open surfaces where the shear stress of the fluid must be zero. This translates to a Neumann-type boundary condition where the gradient of the velocity profile is zero. Examples of this flow case include Couette flow as used, e.g., in plate/cone viscometers. Traditionally, slip-flow is an effect usually studied only at elevated temperatures [2] [3]. However, there are many cases in microfluidics where slip flow occurs. Examples include, e.g., in air-retaining drag-reducing Salvinia-type [4] [5] and superhydrophobic [6] surfaces with [7] or without surface textures [5]. In many of these cases, the flow exhibits mixed boundary conditions with one surface showing slip boundary behaviour whereas the opposing surface shows no-slip boundary behaviour. Deriving analytical solutions for flow cases exhibiting Neumann-type boundary conditions or even mixed boundary conditions is significantly more difficult. These cases are usually studied numerical solver packages [8], lattice-Boltzmann or molecular dynamics simulations [9].

This paper will derive an analytical solution to mixed slip/no-slip boundary conditions in two dimensions in rectangular channel cross-sections. This case is the most common case in microfluidic systems. We will also show that the Microsoft Excel spreadsheet developed for solving no-slip boundary flow scenarios can be adapted to derive the same solution within seconds. This allows deriving solutions to mixed Neumann/Dirichlet boundary condition flow scenarios for a wide variety of cross-sections. 


\section{Rectangular Channel with Two-Dimensional Flow: No-Slip Boundary Conditions Along y-Axis and Mixed Boundary Conditions Along z-Axis}

\subsection{Analytical Solution}

\subsubsection{Simplified Navier-Stokes Equation for Poiseuille Flow}

The fundamental equation for the Poiseuille flow in microfluidics is given by

$$
\Delta \boldsymbol{v}=\frac{1}{\eta} \nabla p
$$

which can be written as

$$
\frac{\partial^{2} v_{x}}{\partial y^{2}}+\frac{\partial^{2} v_{x}}{\partial z^{2}}=\frac{1}{\eta} \frac{\Delta p}{\Delta L}
$$

Details on the derivation can be found elsewhere [1]. Here the velocity $v_{x}$ along the $x$-axis is the dependent variable, $\Delta p / \Delta L$ is the driving pressure drop and $\eta$ is the dynamic viscosity. $y$ is the independent variable along the channel width $W$ and $z$ is the independent variable along the channel height $H$.

\subsubsection{Homogeneous Solution}

The solution to Equation (1) is derived by a separation of variables approach. For this we assume the dependent variable $v_{x}(y, z)=Y(y) Z(z)$ to be composed of two functions $Y(y)$ and $Z(z)$ both of which depend on only one independent variable, respectively. Details on this procedure and the derived solutions can be found elsewhere [10]. We begin by finding the homogeneous solution to Equation (1) according to

$$
\begin{gathered}
\frac{\partial^{2} v_{x}}{\partial y^{2}}+\frac{\partial^{2} v_{x}}{\partial z^{2}}=0 \\
Z \frac{\partial^{2} Y}{\partial y^{2}}+Y \frac{\partial^{2} Z}{\partial z^{2}}=0 \\
\frac{1}{Y} \frac{\mathrm{d}^{2} Y}{\mathrm{~d} y^{2}}=-\frac{1}{Z} \frac{\mathrm{d}^{2} Z}{\mathrm{~d} z^{2}}
\end{gathered}
$$

where we exploited the fact that $Y(y)$ and $Z(z)$ are functions of only one independent variable respectively. Equation (2c) will only be satisfied for arbitrary values of $y$ and $z$ if both sides of Equation (2c) result in a constant. We therefore obtain two ordinary differential equations from Equation (2c) which are given by

$$
\frac{1}{Y} \frac{\mathrm{d}^{2} Y}{\mathrm{~d} y^{2}}=-\lambda_{1}
$$

and

$$
\frac{1}{Z} \frac{\mathrm{d}^{2} Z}{\mathrm{dz}}=-\lambda_{2}
$$

Details on these solutions can be found elsewhere [10]. For Equation (3) we 
have the boundary conditions $Y(y=0)=0$ and $Y(y=W)=0$ (both Dirichlet boundary conditions, no-slip) whereas for Equation (4) we have the boundary conditions $Z(Z=0)=0$ (Dirichlet boundary condition, no-slip) and $\mathrm{d} Z / \mathrm{d} z(z=H)=0 \quad$ (Neumann boundary condition, no-slip). Both Equation (3) and Equation (4) have complex conjugated solutions and therefore the general solutions

$$
Y(y)=C_{0} \sin \left(\sqrt{\lambda_{1}} y\right)+C_{1} \cos \left(\sqrt{\lambda_{1}} y\right)
$$

and

$$
Z(z)=C_{2} \sin \left(\sqrt{\lambda_{2}} z\right)+C_{3} \cos \left(\sqrt{\lambda_{2}} z\right)
$$

Applying the boundary condition $Y(y=0)=0$ to Equation (5) yields $C_{1}=0$. Applying the boundary condition $Y(y=W)=0$ yields $C_{0} \sin \left(\sqrt{\lambda_{1}} W\right)=0$ which is only fulfilled if $\sqrt{\lambda_{1}} W=n \cdot \pi$ from which we derive $\lambda_{1}=(n \pi / W)^{2}$. This yields the eigenfunctions $Y_{n}$ of Equation (5) as

$$
Y_{n}(y)=C_{0} \sin \left(n \pi \frac{y}{W}\right)
$$

and the general solution to Equation (3) as

$$
Y(y)=\sum_{n=1}^{\infty} C_{n} \sin \left(n \pi \frac{y}{W}\right)
$$

Applying the boundary condition $Z(z=0)=0$ to Equation (6) yields $C_{3}=0$. Applying the boundary condition $\mathrm{dZ} / \mathrm{dz}(\mathrm{z}=H)=0$ yields $\sqrt{\lambda_{2}} C_{2} \cos \left(\sqrt{\lambda_{2}} H\right)=0$ which is only fulfilled if $\sqrt{\lambda_{2}} H=(2 m+1) \pi / 2$ from which we derive $\lambda_{2}=((2 m+1) \pi / 2 H)^{2}$. This yields the eigenfunctions $Z_{m}$ of Equation (6) as

$$
Z_{m}(z)=C_{m} \sin \left((2 m+1) \frac{\pi}{2} \frac{z}{H}\right)
$$

and the general solution to Equation (4) as

$$
Z(z)=\sum_{m=0}^{\infty} C_{m} \sin \left((2 m+1) \frac{\pi}{2} \frac{Z}{H}\right)
$$

The homogenous solution of Equation (1) is therefore given by

$$
v_{x}(y, z)=Y(y) Z(z)=\sum_{n=1}^{\infty} \sum_{m=0}^{\infty} C_{n m} \sin \left(n \pi \frac{y}{W}\right) \sin \left((2 m+1) \frac{\pi}{2} \frac{z}{H}\right)
$$

\subsubsection{Inhomogeneous Solution}

Using Equation (9) the inhomogeneous solution is obtained from Equation (1) as

$$
\begin{aligned}
& \sum_{n=1}^{\infty} \sum_{m=0}^{\infty} C_{n m}\left(\left(\frac{n \cdot \pi}{W}\right)^{2}+\left((2 m+1) \frac{\pi}{2 H}\right)^{2}\right) \sin \left(n \pi \frac{y}{W}\right) \sin \left((2 m+1) \frac{\pi}{2} \frac{z}{H}\right) \\
& =\frac{1}{\eta} \frac{\Delta p}{\Delta L}
\end{aligned}
$$

where the right-hand side of Equation (10) (which is constant) must be con- 
verted to a two-dimensional Fourier series in order to derive $C_{n m}$ by coefficient comparison. In general, the Fourier series of a constant $C_{0}$ on the interval $0 \leq \xi \leq A$ is given by

$$
f_{\text {const }}(\xi)=\sum_{p=0}^{\infty} \frac{4 \cdot C_{0}}{(2 p+1) \pi} \sin \left((2 p+1) \pi \frac{\xi}{A}\right)
$$

In two-dimensions with $0 \leq \xi \leq A$ and $0 \leq \varsigma \leq B$ a constant is given by the Fourier series

$$
f_{\text {const }}(\xi, \varsigma)=\sum_{n=0}^{\infty} \sum_{m=0}^{\infty} \frac{16 \cdot C_{0}}{(2 n+1)(2 m+1) \pi^{2}} \sin \left((2 n+1) \pi \frac{\xi}{A}\right) \sin \left((2 m+1) \pi \frac{\varsigma}{B}\right)
$$

The right-hand side of Equation (10) is therefore converted to a two-dimensional Fourier series using Equation (11) and setting $A=W$ and $B=2 H$ in which case we can rewrite Equation (10) to

$$
\begin{aligned}
& \sum_{n=0}^{\infty} \sum_{m=0}^{\infty} C_{n m}\left(\left(\frac{(2 n+1) \cdot \pi}{W}\right)^{2}+\left((2 m+1) \frac{\pi}{2 H}\right)^{2}\right) \\
& \sin \left((2 n+1) \pi \frac{y}{W}\right) \sin \left((2 m+1) \frac{\pi}{2} \frac{z}{H}\right) \\
& =\frac{16}{\eta \cdot \pi^{2}} \frac{\Delta p}{\Delta L} \sum_{n=0}^{\infty} \sum_{m=0}^{\infty} \sin \left((2 n+1) \pi \frac{y}{W}\right) \\
& \sin \left((2 m+1) \frac{\pi}{2} \frac{z}{H}\right)((2 n+1)(2 m+1))^{-1}
\end{aligned}
$$

where we have used the fact that the right-hand side requires only odd values of $n$. We can now determine the missing constants $C_{n m}$ as

$$
C_{n m}=-\frac{16}{\eta \pi^{4}} \frac{\Delta p}{\Delta L}\left((2 n+1)(2 m+1)\left(\left(\frac{2 n+1}{W}\right)^{2}+\left(\frac{2 m+1}{2 H}\right)^{2}\right)\right)^{-1}
$$

in which case the solution to Equation (1) is obtained from Equation (9) and Equation (12) as

$$
\begin{aligned}
v_{x}(y, z)= & -\frac{16}{\eta \cdot \pi^{4}} \frac{\Delta p}{\Delta L} \sum_{n=0}^{\infty} \sum_{m=0}^{\infty} \sin \left((2 n+1) \pi \frac{y}{W}\right) \sin \left((2 m+1) \frac{\pi}{2} \frac{z}{H}\right) \\
& \cdot\left((2 n+1)(2 m+1)\left(\left(\frac{2 n+1}{W}\right)^{2}+\left(\frac{2 m+1}{2 H}\right)^{2}\right)\right)^{-1}
\end{aligned}
$$

where we introduce the channel aspect ratio $r$ as

$$
r=\frac{H}{W}
$$

which allows us to rewrite Equation (13) to

$$
\begin{aligned}
v_{x}(y, z)= & -\frac{16 W^{2}}{\eta} \frac{\Delta p}{\Delta L} \frac{1}{\pi^{4}} \sum_{n=0}^{\infty} \sum_{m=0}^{\infty} \sin \left((2 n+1) \pi \frac{y}{W}\right) \sin \left((2 m+1) \frac{\pi}{2} \frac{z}{H}\right) \\
& \cdot\left((2 n+1)(2 m+1)\left((2 n+1)^{2}+\left(\frac{2 m+1}{2 r}\right)^{2}\right)\right)^{-1}
\end{aligned}
$$

and 


$$
\begin{aligned}
v_{x}(y, z)= & v_{x, \max } \frac{16}{\pi^{4}} \sum_{n=1}^{\infty} \sum_{m=0}^{\infty} \sin \left((2 n+1) \pi \frac{y}{W}\right) \sin \left((2 m+1) \frac{\pi}{2} \frac{z}{H}\right) \\
& \cdot\left((2 n+1)(2 m+1)\left((2 n+1)^{2}+\left(\frac{2 m+1}{2 r}\right)^{2}\right)\right)^{-1}
\end{aligned}
$$

with $v_{x, \max }=-W^{2} \Delta p / \eta \Delta L$.

Equation (13") is shown as a three-dimensional plot in Figure 1(a) normalized to the maximum velocity $v_{x, \max }$. The plot shows the expected zero-gradient profile for $z=H$ while all other boundaries show no-slip behavior and therefore $v_{x}=0$.

\subsection{Numerical Solution}

\subsubsection{Numerical Scheme}

The numerical scheme used to solve Equation (1) is based on a finite difference approach and can be written as

$$
\begin{gathered}
F^{(y, z)}=\frac{1}{4}\left(F^{(y+1, z)}+F^{(y-1, z)}+F^{(y, z+1)}+F^{(y, z-1)}-\frac{h^{2}}{\eta} \frac{\Delta p}{\Delta L}\right) \\
F^{(y, z)}=\frac{1}{4}\left(F^{(y+1, z)}+F^{(y-1, z)}+F^{(y, z+1)}+F^{(y, z-1)}-\Gamma\right)
\end{gathered}
$$

where $F^{(y, z)}$ is the value of the dependent variable in cell $(y, z)$ whereas $F^{(y+1, z)}, F^{(y-1, z)}, F^{(y, z+1)}$ and $F^{(y, z-1)}$ are the values of the dependent variable in the neighboring cell in the positive and negative $y$-direction as well as in the positive and negative $z$-direction, respectively. The constant $\Gamma=0.1 h^{2} \Delta p / \eta \Delta L$ is corrected for the unit $\mathrm{mm} / \mathrm{s}$. This scheme was implemented in Microsoft Excel on a domain consisting of $40 \times 40$ cells. After activating recursive calculations, Microsoft Excel yields the solution to Equation (1). Details on the derivation of the spreadsheet can be found in our previous publication [1].

\subsubsection{Implementing Neumann-Type Boundary Conditions}

In order to replicate the scenario displayed in Figure 1(a) we need to implement

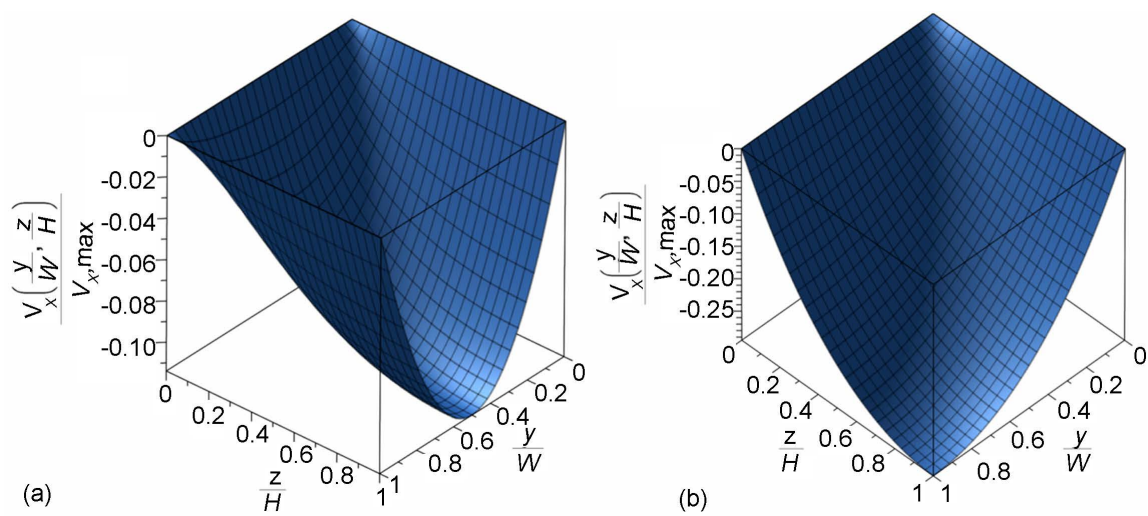

Figure 1. 3D flow profiles. Visualization of the analytical solutions to the flow case discussed in Section 2.1.3 (a) and Section 3.1.2 (b) given by Equation (13") and Equation (20), respectively. The Fourier series have been expanded to $n_{\max }=m_{\max }=50$. These profiles are the assumed analytical solutions used as comparison in Figure 3. 
Neumann-type boundary conditions on our computational domain in the spreadsheet (the used spreadsheet can be found in the supporting information). This can be done by setting the boundary value equal to the value of the neighbouring cell. This effectively implements a Neumann-type boundary condition, i.e., the gradient of the dependent variable will be zero. By adding an offset value according to

$$
=\mathrm{B}\{\text { line number }\}+\text { offset }
$$

the gradient can be set to any desired offset value. For the case shown in Figure 1 (a) we select the cells in the spreadsheet that represent the upper boundary (cells $\mathrm{B} 1$ to AO1). We then link the values of each of these cells to the value of the cell below it, respectively (cells B2 to AO2). We use a pressure gradient of $-0.1 \mathrm{mbar} / \mathrm{mm}$, a channel with a height and width of $100 \mu \mathrm{m}$, respectively, and use water as the fluid in question (viscosity $1 \mathrm{mPa} \cdot \mathrm{s}$ ). After completion of the recursive calculation Figure 2(a) is obtained.

\subsection{Comparison of Analytical and Numerical Solution}

Figure 2(b) shows the numerical output obtained from the spreadsheet in direct comparison with the analytical solution given by Equation (13") using the given values. As can be seen, the error is highest in areas of high gradients, predominantly in the edges of the cross-section. However, the solution should be sufficiently exact for most applications. In order to increase the exactness of the numerical solution, the step width $h$ needs to be reduced further. For this, the resolution of the computational domain must be increased, i.e., the number of cells must be augmented. However, for most applications the given spreadsheet creates sufficiently exact results.

\section{Two-Dimensional Flow Case Mixed Boundary Conditions along $y$-Axis and $z$-Axis}

\subsection{Analytical Solution}

\subsubsection{Homogenous Solution}

In the next step, we extend our discussion to channels with mixed boundary conditions along both channel axes. These types of channels have Dirichlet boundary condition for $y=0$ and $z=0$ (no-slip) Neumann boundary conditions for $y=W$ and $z=H$ (slip). Again, we will first supply an analytical solution to this flow problem beginning with the homogeneous solution to Equation (1). As both $Y(y)$ as well as $Z(z)$ now have to fulfill the same mixed boundary conditions the eigenfunctions will be

$$
Y(y)=\sum_{n=0}^{\infty} C_{n} \sin \left((2 n+1) \frac{\pi}{2} \frac{y}{W}\right)
$$

and

$$
Z(z)=\sum_{m=0}^{\infty} C_{m} \sin \left((2 m+1) \frac{\pi}{2} \frac{z}{H}\right)
$$

which yields the homogeneous solution 


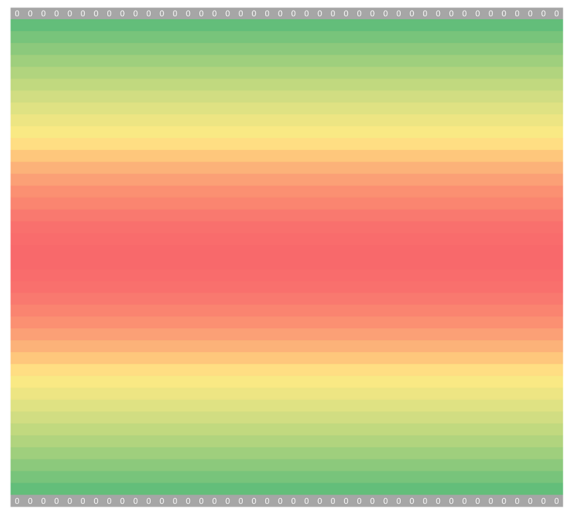

(a)

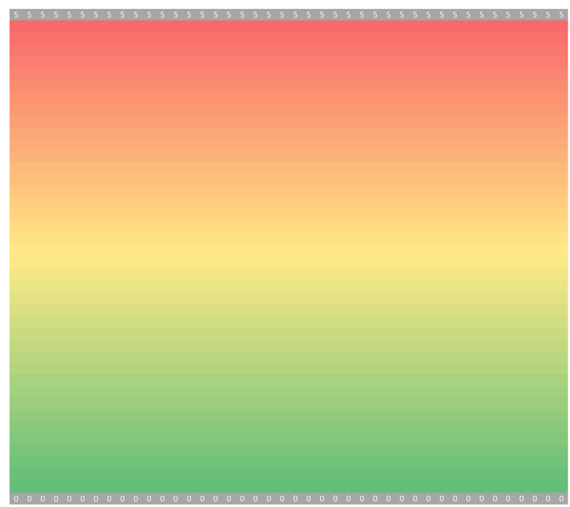

(b)

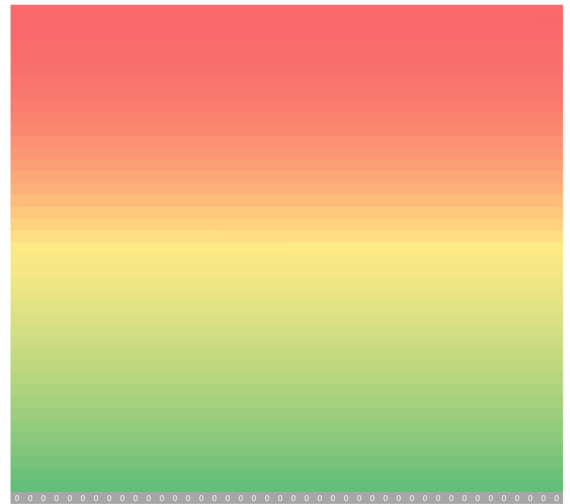

(c)
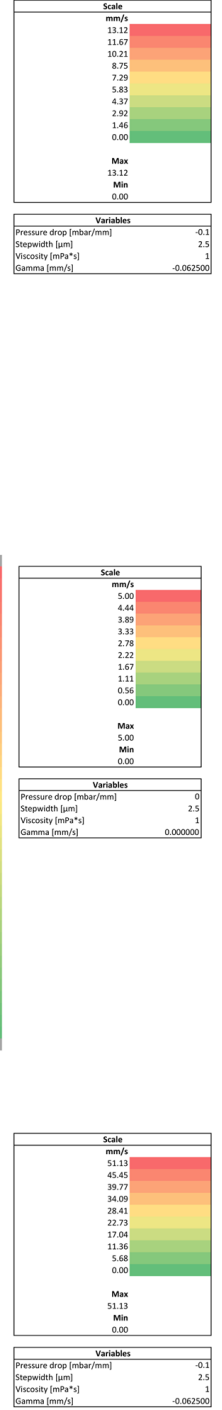

Figure 2. Numerical solutions for different boundary conditions. (a) Numerical output obtained from the Microsoft Excel spreadsheet for solving Equation (1) for cross-section with slip boundary condition (Neumann boundary condition) for $z=H$. (b) Relative error between the numerical output of the spreadsheet and the analytical solution given by Equation (13"). (c) Numerical output obtained from the Microsoft Excel spreadsheet for solving Equation (1) for a cross-section with slip boundary condition for $y=W$ and $z=H$. (d) Relative error between the numerical output of the spreadsheet and the analytical solution given by Equation (20). In all cases a step width $\mathrm{h}$ of $2.5 \mu \mathrm{m}$ was assumed on a domain of $40 \times 40$ cells using water as the fluid. The driving pressure drop was assumed to be $-0.1 \mathrm{mbar} / \mathrm{mm}$ in all cases.

$$
v_{x}(y, z)=Y(y) Z(z)=\sum_{n=0}^{\infty} \sum_{m=0}^{\infty} C_{n m} \sin \left((2 n+1) \frac{\pi}{2} \frac{y}{W}\right) \sin \left((2 m+1) \frac{\pi}{2} \frac{z}{H}\right)(17
$$

\subsubsection{Inhomogeneous Solution}

For the inhomogeneous solution the constant of the right-hand side in Equation 
(1) is again converted to a two-dimensional Fourier series with $0 \leq \xi \leq A$ and $0 \leq \varsigma \leq B$ setting $A=2 W$ and $B=2 H$ in which case we obtain (in analogy to Equation (11b)

$$
f_{\text {const }}(\xi, \varsigma)=\sum_{n=0}^{\infty} \sum_{m=0}^{\infty} \frac{16 C_{0}}{(2 n+1)(2 m+1) \pi^{2}} \sin \left((2 n+1) \pi \frac{\xi}{2 W}\right) \sin \left((2 m+1) \pi \frac{\varsigma}{2 H}\right)
$$

By comparison of coefficients between Equation (17) and Equation (18) $C_{n m}$ can be determined to be

$$
C_{n m}=-\frac{16}{\eta \pi^{4}} \frac{\Delta p}{\Delta L}\left((2 n+1)(2 m+1)\left(\left(\frac{2 n+1}{2 W}\right)^{2}+\left(\frac{2 m+1}{2 H}\right)^{2}\right)\right)^{-1}
$$

in which case the general solution is obtained from Equation (17) as

$$
\begin{aligned}
v_{x}(y, z)= & v_{x, \max } \frac{16}{\pi^{4}} \sum_{n=0}^{\infty} \sum_{m=0}^{\infty} \sin \left((2 n+1) \frac{\pi}{2} \frac{y}{W}\right) \sin \left((2 m+1) \frac{\pi}{2} \frac{z}{H}\right) \\
& \cdot\left((2 n+1)(2 m+1)\left(\left(\frac{2 n+1}{2}\right)^{2}+\left(\frac{2 m+1}{2 r}\right)^{2}\right)\right)^{-1}
\end{aligned}
$$

Equation (20) is shown as a three-dimensional plot in Figure 1(b) normalized to the maximum velocity $v_{x, \max }$. The plot shows the expected zero-gradient profile for $y=W$ and $z=H$ whereas the other boundaries show no-slip behavior.

\subsection{Numerical Solution}

Extending the previous example we will now discuss the flow case with mixed boundary conditions along both the $y$-axis as well as the $z$-axis. This flow case as zero-value Dirichlet boundary conditions for $y=0$ and $z=0$ and zero-value Neumann boundary conditions for $y=W$ and $z=H$. The numerical solution is obtained conveniently by setting all cells of the right-hand side boundary of the domain (cells AP1 to AP45) in the spreadsheet to the neighboring values inside of the domain (cells AO1 to AO45). The values for the step width, the viscosity and the driving pressure drop are not altered. After a couple of seconds the result shown in Figure 2(c) is obtained.

\subsection{Comparison of Analytical and Numerical Solution}

Figure 2(d) shows the relative error between the numerical solution obtained from the spreadsheet and the analytical solution given by Equation (20). As can be seen, the error is virtually non-existing in regions of small gradients, i.e., at the slip boundaries for $y=W$ and $z=H$. Near to the no-slip boundaries the gradient is steepest which is where we find the highest relative errors. Again, in order to reduce the overall error, the domain must be finer discretized, i.e., the number of cells has to be increased and the step width $h$ has to be reduced.

\section{One-Dimensional Flow Cases}

As last examples we will illustrate that the spreadsheet can also be used to obtain 
solutions to one-dimensional flow cases.

Obviously, for all of these cases, analytical solutions exist and are described in the literature. This serves to illustrate that the numerical solutions obtained using the spreadsheet yield correct results also for these cases. The three flow cases addressed are shown in Figure 3.

\subsection{Infinitesimally-Extended Channel Along y-Axis}

The first case is the infinitesimally-extended channel displayed in Figure 3(a). This case is essentially a one-dimensional problem for which Equation (1) simplifies to

$$
\begin{aligned}
& \frac{\partial^{2} v_{x}}{\partial \mathrm{z}^{2}}=\frac{1}{\eta} \frac{\Delta p}{\Delta L} \\
& \frac{\mathrm{d}^{2} v_{x}}{\mathrm{~d} z^{2}}=\frac{1}{\eta} \frac{\Delta p}{\Delta L}
\end{aligned}
$$

where the partial differentials can be converted to ordinary differentials because there is no change along the $y$-axis. The solution to Equation (21) can be obtained by integrating twice using the boundary values $v_{x}(z=0)=0$ and $v_{x}(z=H)=0$ which yields the solution

$$
v_{x}(z)=\frac{1}{2 \eta} \frac{\Delta p}{\Delta L}\left(\left(\frac{z}{H}\right)^{2}-\left(\frac{z}{H}\right)\right)
$$

Compared to the scenarios discussed so far, this scheme is one-dimensional. This requires our two-dimensional spreadsheet to be converted to a one-dimensional problem. Numerically this is implemented by setting the boundary conditions on the left- and right-hand side of our domain from Dirichlet boundary conditions to Neumann boundary conditions. For this select the cells which represent the left-hand side of the boundary (cells A2 to A41 in the provided spreadsheet) and set them equal to the value of the right-hand side cell, respectively, by inserting the formula

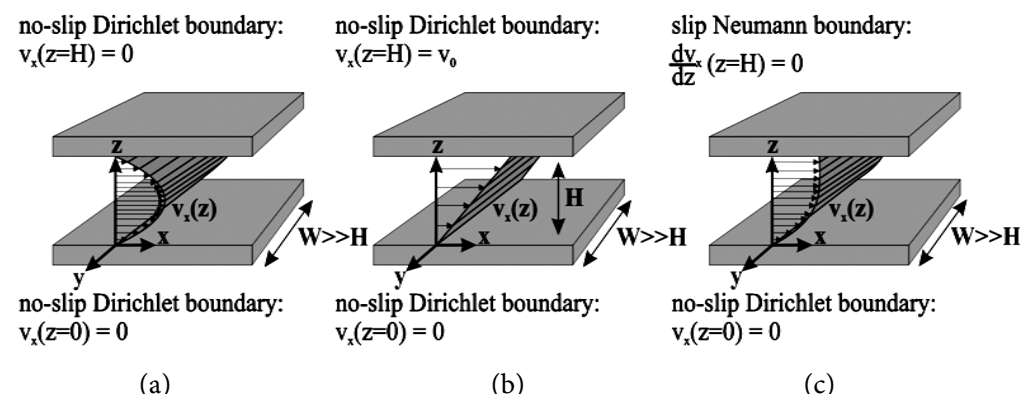

(a)

(b)

(c)

Figure 3. Schematics of one-dimensional flow cases discussed. (a) Infinitesimally-extended channel along the $y$-axis (Dirichlet boundary conditions for top and bottom boundary, zero boundary value for top and bottom boundary). (b) Couette flow (Dirichlet boundary conditions for top and bottom boundary, non-zero boundary value for top boundary, zero boundary value for bottom boundary). (c) Mixed boundary value case (Neumann boundary condition for top boundary, Dirichlet boundary condition for bottom boundary, zero boundary values for top and bottom boundary). 


$$
=\mathrm{B}\{\text { line number }\}
$$

Likewise we select the cells of the right-hand side of the domain (cells AP2 to AP41 in the provided spreadsheet) and set them equal to the neighboring cells within the domain by inserting the formula

$$
=\mathrm{AO}\{\text { line number }\}
$$

This effectively removes the second dimension from the numerical problem. Figure 4(a) shows the numerical solution displayed by the spreadsheet whereas Figure 4(b) shows the direct comparison between the numerical and the analytical solutions which show an exact match.

\subsection{Couette Flow}

The next flow scenario discussed is the one-dimensional Couette flow (see Figure 3(b)). For this scenario we again use Neumann boundary conditions along
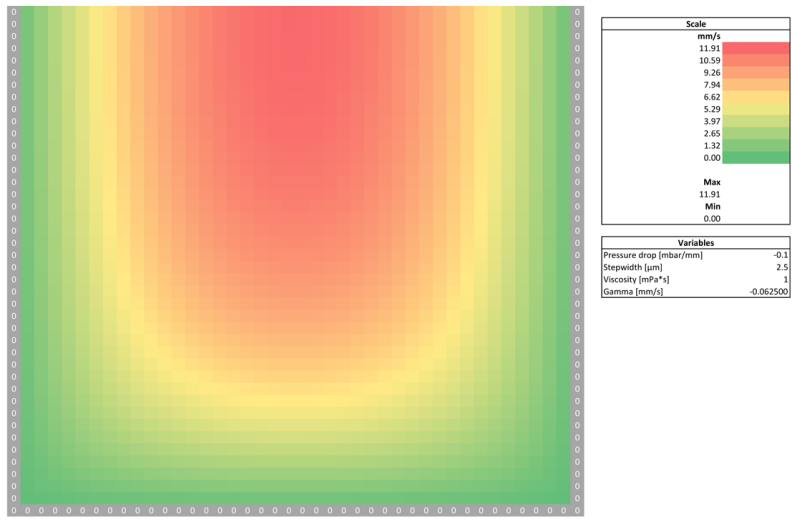

(a)

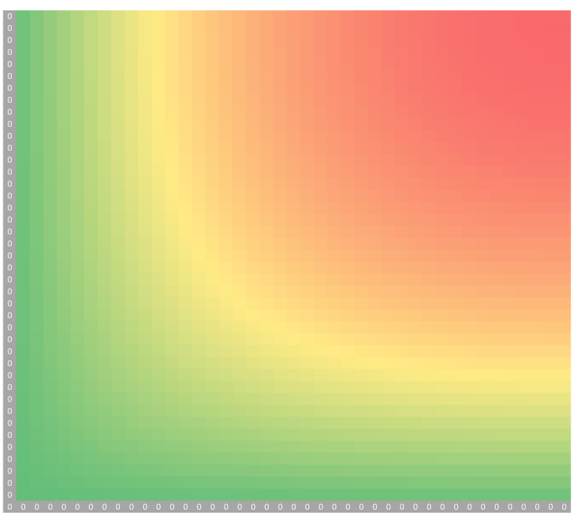

(b)

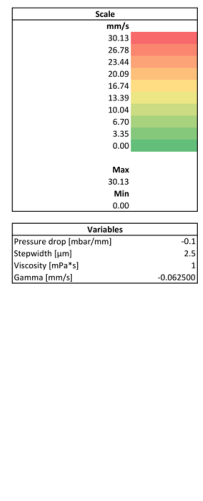

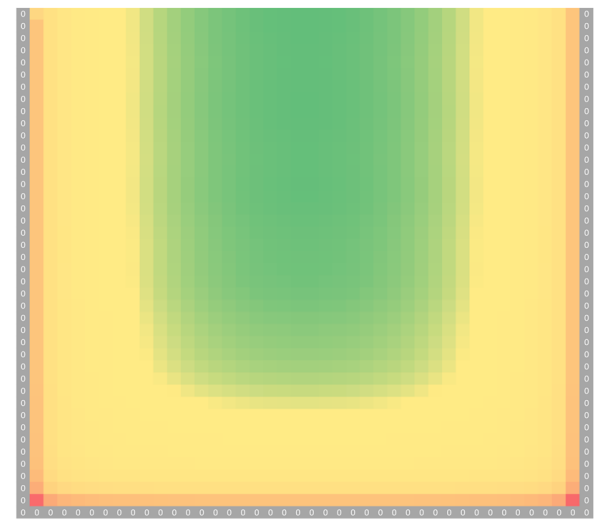

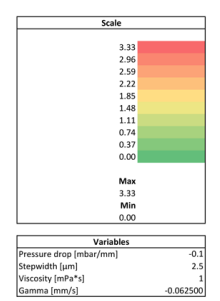

(c)
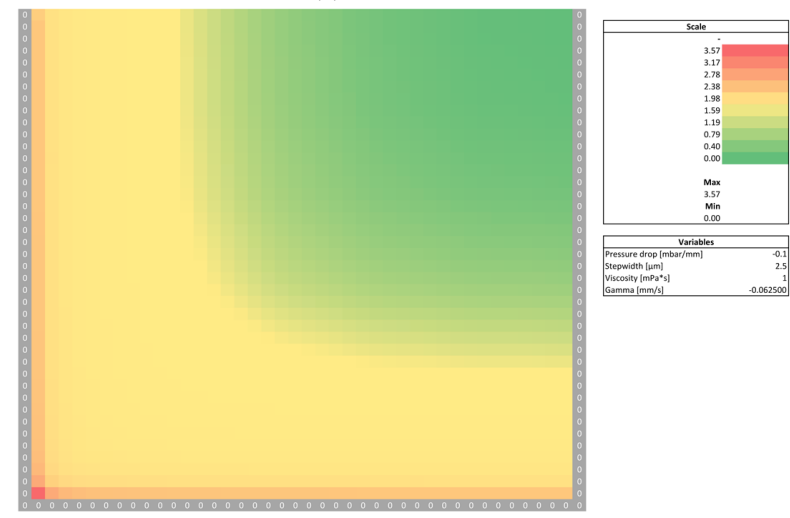

(d)

Figure 4. Numerical solutions for one-dimensional flow cases. Numerical output obtained from the Microsoft Excel spreadsheet for solving Equation (1) for the three one-dimensional flow cases shown in figure 3 (a: Infinitesimally-extended channel; b: Couette flow; c: Mixed boundary conditions with no-slip condition for $\mathrm{z}=0$ and slip condition for $\mathrm{z}=\mathrm{H}$ ). The color-coded outputs shown in $(\mathrm{a} / \mathrm{c} / \mathrm{e})$ are the velocity profiles obtained from a microfluidic flow in a channel with $100 \mu \mathrm{m}$ height. The flow in (a/e) is driven by a constant pressure drop of $-0.1 \mathrm{mbar} / \mathrm{mm}$ whereas the flow in (c) is driven by a moving top boundary. All cases use water as fluid. The step width $\mathrm{h}$ of the numerical scheme was set to $2.5 \mu \mathrm{m}$, i.e., each cell represents a cross-section of $2.5 \times 2.5 \mu^{2}$. (b/d/f) Comparison of the numerically obtained solutions (diamonds) with the analytical solutions (solid line) calculated using Equation (22) (b), Equation (24) (d) and Equation 25 (f), respectively. As can be seen the results are in good agreement. 
the $y$-axis therefore reducing the flow scenario to one dimension. In comparison to Poiseuille flow, Couette flow is not driven by a pressure drop along the channel axis but by a movement of one of the boundaries (in our case the top boundary). In comparison to the flow scenarios discussed so far, Couette flow requires a non-zero Dirichlet boundary for $z=H$. This can be done in the spreadsheet by simply setting the values of the top boundary (cells A1 to AO1) to a given value $v_{o}$. The analytical solution for Couette flow is derived from Equation (21b) after setting the right-hand side to zero (as there is no driving pressure drop) and integrating twice to find

$$
v_{x}(z)=C_{1} z+C_{2}
$$

With the boundary conditions $v_{x}(z=0)=0$ and $v_{x}(z=H)=v_{o}$ the solution is obtained to be

$$
v_{x}(z)=v_{o} \frac{z}{H}
$$

In the example in the spreadsheet we use $v_{o}=5 \mathrm{~mm} / \mathrm{s}$. Setting the values of the cells of the upper boundary (cells A1 to AP1) in the spreadsheet to this value results in the velocity profile shown in Figure 4(c). Figure 4(d) shows the analytical solution alongside the numerical solution which shows that, again, the correct numerical solution is obtained.

\subsection{One-Dimensional Mixed Boundary Condition}

As a third example, we will use a one-dimensional flow scenario with mixed boundary conditions along the $y$-axis (see Figure $3(\mathrm{c})$ ). In this example, the lower boundary of the domain has a zero-value Dirichlet boundary (no-slip) whereas the upper boundary has a zero-value Neumann boundary (slip). This is the one-dimensional version of the case discussed in Section 3. Again we use Neumann boundary conditions on the left- and right-hand side boundaries of the domain therefore obtaining a one-dimensional flow. We set the value of the top boundary (cells A1 to AO1) equal to the values of the neighboring cell inside of the domain, respectively (cells A2 to AO2). This implements a Neumann boundary condition at the top of the domain. Again we use a pressure gradient of $-0.1 \mathrm{mbar} / \mathrm{mm}$, a channel height of $100 \mu \mathrm{m}$ and water as the fluid in question (viscosity $1 \mathrm{mPa} \cdot \mathrm{s}$ ). After completion of the recursive calculation the output shown in Figure 4(e) is obtained.

The analytical solution to this flow scenario can be obtained by applying the boundary conditions $v_{x}(z=0)=0$ and $\left(\mathrm{d}_{x}\right) / \mathrm{d} z(h=H)=0$ to Equation (23) which yields the solution

$$
v_{x}(z)=\frac{1}{\eta} \frac{\Delta p}{\Delta L}\left(\frac{1}{2}\left(\frac{z}{H}\right)^{2}-\left(\frac{z}{H}\right)\right)
$$

The direct comparison between the numerical and the analytical solution is shown in Figure 4(f). As can be seen the numerical solution is again, close-toexact. 


\section{Conclusion}

In this paper, we extended the concept of using a spreadsheet analysis tool such as Microsoft Excel to solve the fundamental equation for Poiseuille flow, i.e., the simplified Navier-Stokes equation in arbitrary cross-sections in one and two dimensions implementing different boundary conditions. Besides zero-value and fixed-value Dirichlet boundary conditions (which are commonly used for implementing no-slip boundaries), we showed that simple modifications to the spreadsheet are sufficient to implement Neumann boundary conditions which set the gradient of the velocity profile to fixed values. These boundaries are required to implement slip boundaries which are commonly encountered on open surfaces or air-retaining substrates. We have shown that the solutions obtained within seconds from the spreadsheet compare very well to the analytical solutions obtained for three cases of one-dimensional flows: infinitesimally-extended channel, Couette flow and one-dimensional mixed boundary condition flow. In terms of two dimensional flows we provided analytical solutions to the case of a no-slip/no-slip boundary pair along the y-axis and a no-slip/slip boundary pair along the $z$-axis as well as a solution to the case of a no-slip/slip boundary pair along both the $y$-and the $z$-axis. In both cases, the numerical solutions obtained agree well with the analytical solutions. This underlies the potential of using simple-to-use spreadsheet analysis tools such as Microsoft Excel to derive important features such as the velocity profiles in arbitrary channel cross-sections instead of referring to expensive and difficult-to-use numerical solver packages. As we have shown this approach copes very well with different and even mixed boundary conditions and provides solutions within seconds even in cases where analytical solutions are rather difficult to derive.

\section{Acknowledgements}

This work was funded by the Bundesministerium für Bildung und Forschung (BMBF), funding code 03X5527 "Fluoropor".

\section{References}

[1] Richter, C., Kotz, F., Giselbrecht, S., Helmer, D. and Rapp, B. E. (2016) Numerics Made Easy: Solving the Navier-Stokes Equation for Arbitrary Channel Cross-Sections Using Microsoft Excel. Biomedical microdevices, 18, 1-8. https://doi.org/10.1007/s10544-016-0070-2

[2] Ngoma, G.D. and Erchiqui, F. (2007) Heat Flux and Slip Effects on Liquid Flow in a Microchannel. International Journal of Thermal Sciences, 46, 1076-1083. https://doi.org/10.1016/j.ijthermalsci.2007.02.001

[3] Yu, S. and Ameel, T.A. (2001) Slip-Flow Heat Transfer in Rectangular Microchannels. International Journal of Heat and Mass Transfer, 44, 4225-4234.

[4] Barthlott, W., Schimmel, T., Wiersch, S., Koch, K., Brede, M., Barczewski, M. et al. (2010) The Salvinia Paradox: Superhydrophobic Surfaces with Hydrophilic Pins for Air Retention Under Water. Advanced Materials, 22, 2325-2328. https://doi.org/10.1002/adma.200904411

[5] Kavalenka, M.N., Vuillers, F., Lischker, S., Zeiger, C., Hopf, A., Rohrig, M., et al. 
(2015) Bioinspired Air-Retaining Nanofur for Drag Reduction. ACS Applied Materials \& Interfaces, 7, 10651-10655.

[6] Wang, C., Tang, F., Hao, P., Li, Q. and Wang, X. (2016) Experimental Study on the Drag Reduction Effect of a Rotating Superhydrophobic Surface in Micro Gap Flow Field. Microsystem Technologies, 1-8. https://doi.org/10.1007/s00542-016-3097-7

[7] Quéré, D., Lafuma, A. and Bico, J. (2003) Slippy and Sticky Microtextured Solids. Nanotechnology, 14, 1109. https://doi.org/10.1088/0957-4484/14/10/307

[8] Hlushkou, D., Kandhai, D. and Tallarek, U. (2004) Coupled Lattice-Boltzmann and Finite-Difference Simulation of Electroosmosis in Microfluidic Channels. International Journal for Numerical Methods in Fluids, 46, 507-532. https://doi.org/10.1002/fld.765

[9] Priezjev, N.V. (2007) Rate-Dependent Slip Boundary Conditions for Simple Fluids. Physical Review E, 75, p. 051605.

[10] Rapp, B.E. (2016) Microfluidics: Modeling, Mechanics and Mathematics. Vol. 1, Elsevier, Cambridge.

Submit or recommend next manuscript to SCIRP and we will provide best service for you:

Accepting pre-submission inquiries through Email, Facebook, LinkedIn, Twitter, etc. A wide selection of journals (inclusive of 9 subjects, more than 200 journals)

Providing 24-hour high-quality service

User-friendly online submission system

Fair and swift peer-review system

Efficient typesetting and proofreading procedure

Display of the result of downloads and visits, as well as the number of cited articles

Maximum dissemination of your research work

Submit your manuscript at: http://papersubmission.scirp.org/

Or contact jbise@scirp.org 